\title{
Flaw Tolerance of Nuclear Intermediate Filament Lamina under Extreme Mechanical Deformation
}

\author{
Zhao Qin $^{\dagger}$ and Markus J. Buehler ${ }^{\dagger, t, \S, *}$ \\ ${ }^{\dagger}$ Laboratory for Atomistic and Molecular Mechanics (LAMM), Department of Civil and Environmental Engineering, ${ }^{\ddagger}$ Center for Computational Engineering, and \\ ${ }^{\S}$ Center for Materials Science and Engineering, Massachusetts Institute of Technology, 77 Massachusetts Avenue, Cambridge, Massachusetts 02139, United States
}

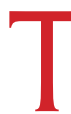
The nuclear lamina is a dense meshwork at the inner membrane of the nuclear envelope of eukaryotic cells ${ }^{1}$ and is formed primarily by $\sim 10 \mathrm{~nm}$ wide type- $V$ intermediate filaments composed of lamin proteins. ${ }^{1-4}$ The lamina contributes largely to the structural integrity of the nucleus, and therefore a key biological role is to protect genetic material against extreme environmental conditions. ${ }^{5,6}$ The lamina is broken down during mitosis; this, however, represents only a relatively short period during the life of a cell., ${ }^{7,8}$ Emphasizing the importance of this meshwork is the fact that there are at least 13 human diseases associated with mutations in lamin proteins. For example, the permanent loss of lamin proteins causes muscular dystrophy and cardiomyopathy, and overaccumulation of lamin mutants at the nuclear membrane causes the rapid aging disorder Hutchinson-Gilford progeria syndrome (HGPS). ${ }^{9-11}$

What is remarkable is that all the various types of nuclear lamina naturally feature structural imperfections, defects, and heterogeneities including those induced by nuclear pores. ${ }^{12-14}$ Defects in the lamina can also be caused by localized forces from cytoskeletal structures or by atomic-scale defects due to the imperfect assembly of the meshwork, leading to void formation in the meshwork. ${ }^{1,12,13,15}$ Another source of imperfections is generated by the clustering of lamin-associated nuclear pore complexes, which can grow rather large and are naturally present in virtually all cells. ${ }^{13,14,16}$ Other possible factors include ionizing radiation that results in the breakage of covalent or noncovalent bonds within protein structures and thus results in structural defects. ${ }^{17-19}$ It has been shown that continuous radiation, as experienced for example during space flight, causes cracks to occur around nuclear pores
ABSTRACT The nuclear lamina, composed of intermediate filaments, is a structural protein meshwork at the nuclear membrane that protects genetic material and regulates gene expression. Here we uncover the physical basis of the material design of nuclear lamina that enables it to withstand extreme mechanical deformation of $>100 \%$ strain despite the presence of structural defects. Through a simple in silico model we demonstrate that this is due to nanoscale mechanisms including protein unfolding, alpha-to-beta transition, and sliding, resulting in a characteristic nonlinear force - extension curve. At the larger microscale this leads to an extreme delocalization of mechanical energy dissipation, preventing catastrophic crack propagation. Yet, when catastrophic failure occurs under extreme loading, individual protein filaments are sacrificed rather than the entire meshwork. This mechanism is theoretically explained by a characteristic change of the tangent stress -strain hardening exponent under increasing strain. Our results elucidate the large extensibility of the nuclear lamina within muscle or skin tissue and potentially many other protein materials that are exposed to extreme mechanical conditions, and provide a new paradigm toward the de novo design of protein materials by engineering the nonlinear stress-strain response to facilitate flaw-tolerant behavior.

KEYWORDS: nuclear lamina $\cdot$ nuclear envelope breakdown · intermediate filament biological material $\cdot$ multiscale modeling $\cdot$ flaw tolerance $\cdot$ materiomics

which leads to a complete fragmentation of nuclear envelopes once a critical defect concentration is reached ${ }^{17}$ (see Figure S2 in the Supporting Information).

While experimental data has provided clear evidence that nuclei in healthy cells can effectively withstand extreme dilations and deformation without rupture, ${ }^{15}$ conventional material models have failed to explain their significant capacity to expand without failure. This is because in most existing models locations of structural imperfections such as crack-like inclusions present singularities for stresses, which typically causes a deterioration of material properties. However, this is not observed in nuclear lamina which provides an extremely robust mechanical framework to protect genetic material. ${ }^{20}$ Here we present an answer to this intriguing question and explain a general mechanism by which the intermediate filament meshwork, and *Address correspondence to
mbuehler@MIT.EDU.

Received for review January 11, 2011 and accepted March 8, 2011.

Published online March 08, 2011

(C) 2011 American Chemical Society $10.1021 / \mathrm{nn} 200107 \mathrm{u}$ 
potentially other protein materials, are capable of forming an extremely robust flaw-tolerant material. We use a simple model developed based on a multiscale approach to assess the mechanisms of defect initiation and propagation in the nuclear lamina under extreme loading conditions, mimicking the conditions a cell experiences during tissue stretching and contraction. The use of a multiscale approach to deal with this problem allows us to simulate a system with tens of micrometers dimension while retaining information about molecular structures and mechanisms.

\section{RESULTS AND DISCUSSION}

Computational Modeling. We consider a meshwork model resembling a Xenopus oocyte nuclear lamina ${ }^{1,12}$ because the structure and mechanical properties of this meshwork are experimentally well characterized, and it represents one of the few measured systems of a pure nuclear lamina without mechanical contributions of chromatin. ${ }^{13,15}$ It is noted that so far only amphibian oocytes' nuclear lamina has been shown to form a rather regular lattice, ${ }^{1,13,15}$ while the structure of nuclear lamina in other cells is likely more disordered and more tightly associated with chromatin. ${ }^{13}$ Thus it is important to note that the model we study here may only represent a first approximation to the structure of other nuclear laminas. Figure 1a illustrates the hierarchical structure of this nuclear lamina. ${ }^{1,3,12,21} \mathrm{We}$ consider the meshwork with a small crack-like defect using uniaxial and biaxial tension (see Figure $1 \mathrm{~b}$, where the meshwork geometry is given in Figure 1c, with a characteristic defect size $a$ ) to mimic in situ loading conditions. For instance, in vivo pressure differences across the nuclear envelope can result in an exposure of the nuclear lamina to significant biaxial tension. ${ }^{15}$ As demonstrated by earlier experimental and computational molecular mechanics studies of the single intermediate filament level, the deformation mechanism is characterized first by elastic elongation, $\alpha$ helix unfolding, ${ }^{22}$ alpha-to-beta transition, ${ }^{23}$ and eventual stick-slip-sliding ${ }^{24}$ (Figure 1d; for details see Materials and Methods). This results in the characteristic nonlinear force-extension of a single fibril as shown in Figure $1 d$, with a severe change in the tangent modulus as strain is increased. As a model we use the mechanical response of vimentin intermediate filaments obtained in the earlier work ${ }^{21}$ for exploring the mechanics of lamin meshwork. This is a reasonable first approximation because all intermediate filament proteins consist of tightly packed and aligned subunits, where each subunit is primarily composed of an extended coiled-coil structure which is crucial for the molecular mechanical properties as described above. ${ }^{25}$ Indeed a direct comparison between lamin and vimentin molecular mechanics reveals that the mechanical response of the lamin dimer is found to be similar as the vimentin dimer (see Figure S4 in Supporting
Information). It has been shown in earlier works that this force-extension result agrees with experimental measurements for the general class of intermediate filament protein family ${ }^{21}$ and we thus consider this in the formulation of our mesoscopic model to mimic the basic quasi-static deformation properties at vanishing pulling rates as relevant for physiological and experimental deformation speeds. This is an idealized mechanics model, which, although being limited by missing the diversity of intermediate filament types, offers a powerful starting point for understanding the fundamentals of the mechanical properties of intermediate filament meshworks. We emphasize that the goal of our model is not to accurately reflect the mechanical properties of one specific or all the different types of nuclear laminas. Rather it is formulated deliberately as a simple model to study generic aspects of the mechanical properties of protein meshwork materials at the microscale relating to the underlying molecular mechanics.

The Flaw Tolerance of Nuclear Lamina. On the basis of the knowledge of the behavior of a single fibril we now assess the mechanical response of an entire meshwork to large deformation by applying increasing uniaxial tension at the top and bottom layers. Figure 2a shows snapshots of the meshwork model with increasing deformation. We observe that no catastrophic failure of the meshwork occurs below $161 \%$ strain. However, we find that the crack geometry transforms drastically from an initial sharp edge in the $x$-direction into an elliptic shape, where the longest axis pointed in the $y$ direction, with a ratio between long axis and short axis being $\sim 3$ (Figure 2a). What is remarkable is that instead of forming a rather small localized deformation zone at the crack tip as seen in linear elastic materials such as concrete, carbon fibers, or silica, virtually all filaments in the meshwork (oriented in the $y$-direction) undergo large deformation and unfold. This represents the formation of a very large "yield" region in which the elastic energy supplied by applied forces is dissipated. A detailed analysis of the forces that act on those filaments indeed confirms that the coiled-coil structure within each molecular subunit has undergone secondary structure unfolding via the breaking of clusters of hydrogen bonds up to strains of $90 \%$. As the load is increased beyond $90 \%$ strain, a greater number of filaments begin to stiffen due to the onset of an alpha-beta transition at the molecular scale. For even greater loads, and when deformation reaches $161 \%$, we observe that deformation starts to localize at the crack tip, resulting in eventual catastrophic failure of the meshwork. Under catastrophic failure the breakdown of the meshwork is mediated by highly localized breaking of individual filaments at the tip of the crack, and at the molecular scale due to slip of individual protein chains against each other (Figure 2a, snapshot iv). 
a

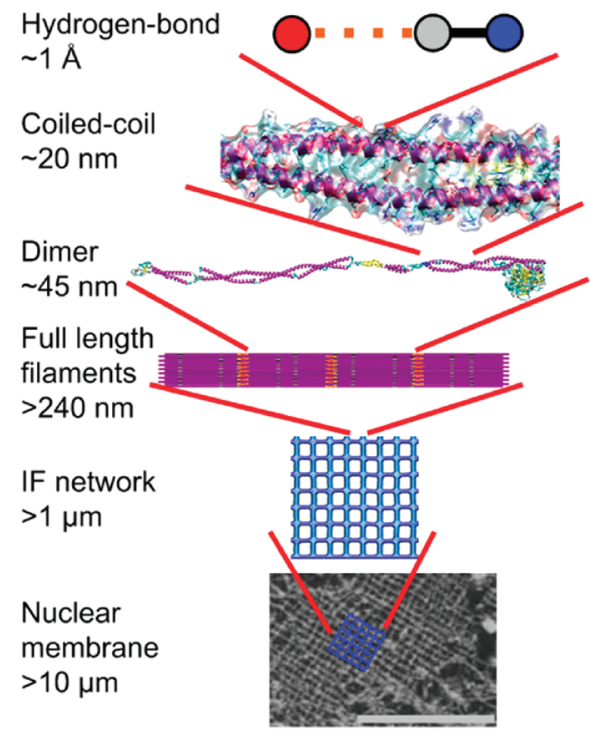

b

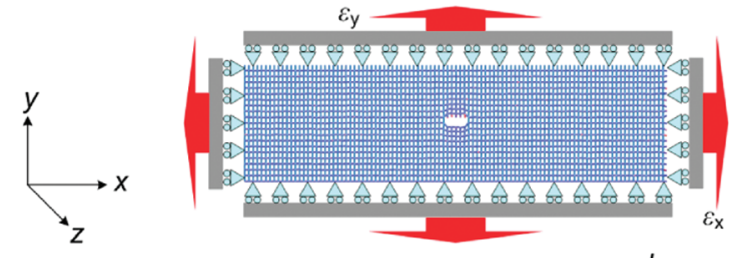

C
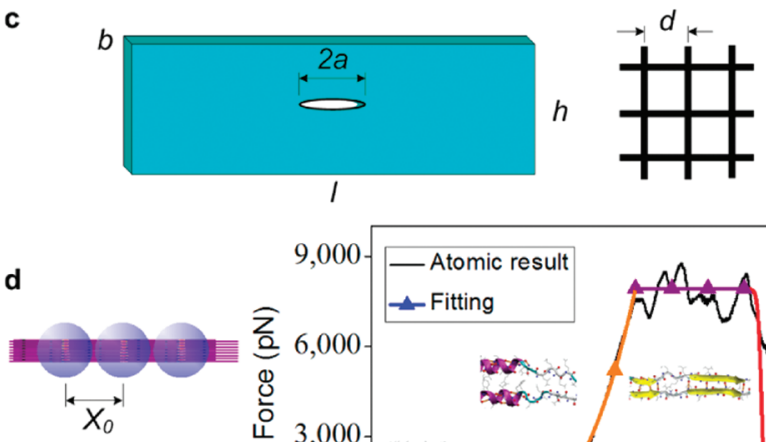

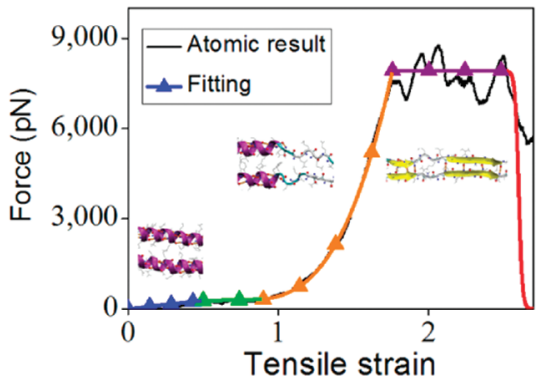

Figure 1. Hierarchical structure of nuclear lamina as found in the inner layer of nuclear envelop of Xenopus oocytes, boundary conditions in calculations, geometry of the meshwork model, and mechanical properties of a single intermediate filament. (a) Schematic of the hierarchical structure of nuclear laminas that ranges from nanoscale to macroscale. The image shows typical structural features of nuclear laminas, including hydrogen bonds, coiled-coil composed of alpha-helices, a lamin A dimer composed of coiled-coil and linkers, bundles of dimers that fuse laterally to form full length filaments with a diameter of $\sim 10 \mathrm{~nm}$, which form an orthogonal structural meshwork attached to the inner layer of nuclear envelope with a lattice constant of $\sim 50 \mathrm{~nm}^{1}$. The scale bar in the nuclear membrane image is $1 \mu \mathrm{m}$. (b) The orthogonal meshwork model, boundary conditions, and the coordinate system used in our simulations. The initial crack is oriented in the $x$-direction and the meshwork is loaded in one direction (typically the $y$-direction) or in both directions. The consideration of different loading conditions mimics the randomness of the deformation field relative to crack directions in nuclear laminas. (c) The geometry parameters of the meshwork model. The parameters $b, l$, and $h$ are the thickness, length, and width of the meshwork model, $d$ is the lattice constant $(\sim 50 \mathrm{~nm})$ and $2 a$ is the initial crack length. (d) Schematic of the coarse-graining method with which each filament is modeled. A collection of mesoscopic beads are used to model the full length filament, where the equilibrated nearest-neighbor bead-to-bead distance is $X_{0}$, and a nonlinear interbead potential obtained from stretching tests of the full atomic intermediate filament model. No molecular unfolding is observed for $\varepsilon<50 \%, \alpha$ helix unfolding is observed as $\varepsilon \geq 50 \%$, a alpha-to-beta transition is observed as $\varepsilon \geq 90 \%$, and beta-sheets slide against each other beyond $\varepsilon \geq 180 \%$ and filaments fail at $\varepsilon \geq 260 \%$ strain. Inserted figures are snapshots of the atomic model of a segment of the 2B domain of the intermediate filament dimer in stretching to visualize molecular mechanisms of deformation.

To summarize our observations, we find a sequence of events that involves delocalization of deformation at applied strains below $161 \%$ strain, followed by localization of deformation at larger applied strains and eventual catastrophic failure during which a small number of filaments break. The ability of the material to mitigate the effect of the defect by dissipating energy via unfolding of all filaments in the meshwork is referred to as flaw tolerance. This flaw tolerance can be theoretically explained by the characteristic nonlinear material behavior provided by the intermediate filament meshwork. The stress-strain curves for the bulk material (with and without defect) are depicted in Figure $2 \mathrm{~b}$ and the Young's modulus $(E)$ vs strain $\left(\varepsilon_{y}\right)$ relation depicted in Figure $2 \mathrm{c}$ show that the meshwork features a highly nonlinear deformation character, with initial softening, followed by stiffening and eventual softening at the failure point. The lowest tangent modulus $E$ is obtained at $70 \%$ strain and features a 3 -fold decrease compared with the initial value at zero strain. The tangent modulus $E$ then significantly increases with strain, and at the failure point reaches a value that is 24fold as large compared to its initial value.

The Mechanism of Flaw Tolerance in the Nuclear Lamina. The bulk strain-stress relation of a general nonlinear (that is, hyperelastic) material can be given by a simple power law as $\sigma \sim \varepsilon^{N}$, where $N$ is the so-called hardening exponent that describes the stress-strain response of a material. Thereby, $N<1$ denotes softening behavior (also referred to as "elastic-plastic"), and $N>1$ represents a stiffening material. As seen directly in Figure 1d the behavior of the protein meshwork is therefore characterized by a change in the local hardening exponent $N$ as the loading is increased, where for deformation up to $90 \%, N=0$, and for larger deformation of up to $176 \%$ strain, $N \gg 1$.

Why is this important? It is known from continuum fracture theory that crack-like defects such as voids typically result in stress and strain concentrations, which are mathematically described as singularities of stresses at the tip of the crack. The stress and strain singularity at the crack tip of a nonlinear material is 
a
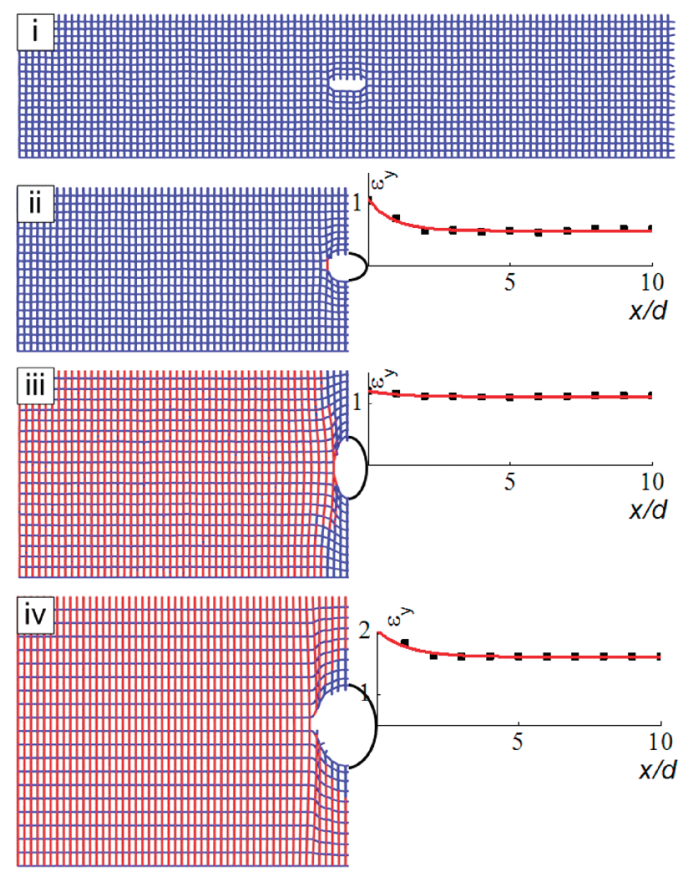

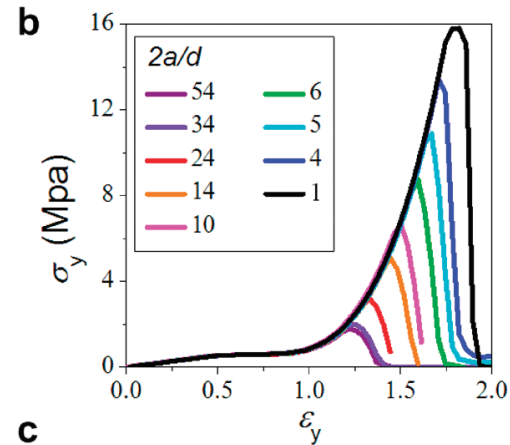

C

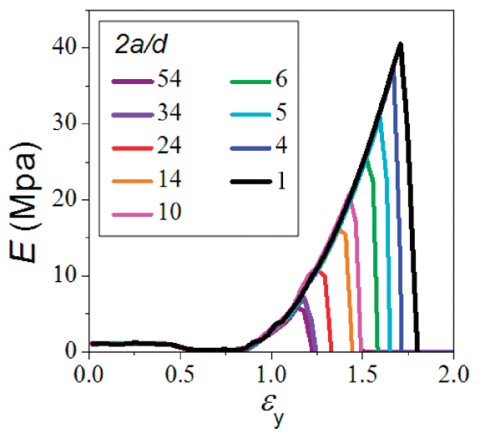

Figure 2. Deformation of the nuclear lamina under uniaxial loading at different levels of applied strain and mechanical analysis of the stress - strain properties of a meshwork with different crack sizes. (a) The snapshots $i$-iv show the deformation field for different levels of applied strain: i, $\varepsilon=38 \%$; ii, $\varepsilon=58 \%$; iii, $\varepsilon=110 \%$; and iv, $\varepsilon=161 \%$. Each filament is colored according to its structural character, with blue denoting the structure before the alpha-to-beta transition has occurred, and red denoting the structure after the alpha-to-beta transition has occurred (at filament strains of around $90 \%$ ). Tensile strains of filaments in the $x$-direction starting from the crack tip are plotted for snapshots ii-iv, where snapshot iv shows the onset of propagation of the crack. (b) Stress-strain relation for meshwork models with different initial crack lengths. (c) Tangent modulus-strain relation derived from panel $b$.

given by ${ }^{26}$

$$
\frac{\sigma_{\mathrm{C}}}{\sigma_{\mathrm{F}}}=F a^{N /(1+N)} \quad \text { and } \quad \frac{\varepsilon_{\mathrm{C}}}{\varepsilon_{\mathrm{F}}}=F^{1 / N} a^{1 /(1+N)}
$$

where $\sigma_{\mathrm{C}}$ and $\varepsilon_{\mathrm{C}}$ represent the critical structural transition or failure stress and strain of a single filament at the crack tip, $\sigma_{\mathrm{F}}$ and $\varepsilon_{\mathrm{F}}$ are the applied stress and strain to the meshwork to reach $\sigma_{\mathrm{C}}$ and $\varepsilon_{\mathrm{C}}$ at the crack tip, and $F=\left[K /\left(\sqrt{ } \pi \sigma_{\mathrm{C}}\right)\right]^{-[2 N /(1+N)]}$ is a material constant $(K$ is the material's fracture toughness). ${ }^{27}$ The crack length, $a$, relates to size of the imperfections in the structure (see for example, in Figure 1c). Equation 1 shows that the nature of the stress or strain concentration depends strongly on the hardening exponent $N$.

We now use this simple model of how stresses and strains are distributed near a crack to explain the findings from our simulations. The first important insight used here is that the stress-strain response of the material in different regimes of deformation can be approximated by different values of $N$, reflecting the behavior seen in Figure 2b,c. Equation 1 predicts that a change in $N$ leads to a drastic change in the stress and strain distribution near a crack tip. Specifically, $N=0$ leads to a highly delocalized stress field but a localized strain field, where $N \gg 1$ leads to a highly localized stress field and a delocalized strain field. We note that here, "localized" means that a quantity is larger at the crack tip than elsewhere, and "delocalized" means that a quantity is of similar magnitude at the crack tip than elsewhere in the system.

Indeed, in agreement with the notion that the hardening exponent $N$ changes as the meshwork is deformed, we observe two distinct types of stress and strain distributions around the crack tip. First, in the softening regime of up to $90 \%$ strain, the strain of the highly stretched individual filament at the crack tip tends to reach the end of the softening stage of $90 \%$ and leaves filaments further away from the crack tip exposed to a much smaller (applied) strain of $58 \%$ (as shown in Figure $2 \mathrm{a}(\mathrm{ii}))$, and thus $\varepsilon_{\mathrm{C}} / \varepsilon_{\mathrm{F}}=1.55$. This represents a localized distribution of strain, where all filaments oriented in the $y$-direction feature strains between those two bounds. However, because of the plateau in the force-strain curve the tensile forces that act on each filament for this range of strains are the same as shown in Figure $1 \mathrm{~d}$, implying that the stress is delocalized and $\sigma_{\mathrm{C}} / \sigma_{\mathrm{F}} \approx 1$. This corresponds to the situation when $N \rightarrow 0$ (reflecting softening) in eq 1 , which agrees with the hypothesis that this case leads to a delocalized stress distribution.

Second, in the stiffening stage from $90 \%$ up to $176 \%$ strain, we observe that the strain of filaments at the crack tip is $120 \%$, and filaments further away from the crack tip are exposed to an applied strain of $110 \%$ (as shown in Figure 2a(iii)). While the strain distribution is delocalized, quantified by $\varepsilon_{\mathrm{C}} / \varepsilon_{\mathrm{F}} \approx 1$, it 
iv

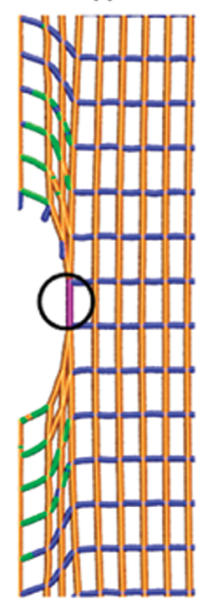

V

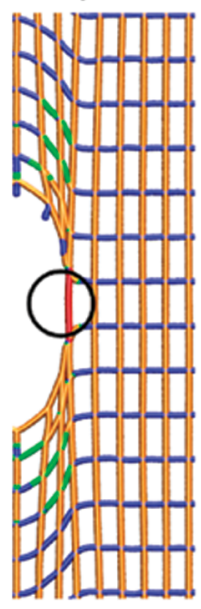

b
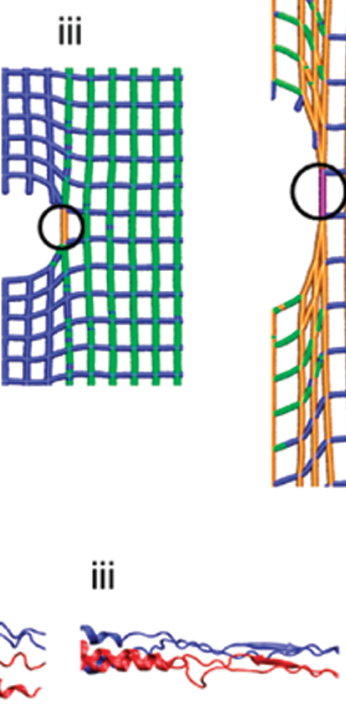

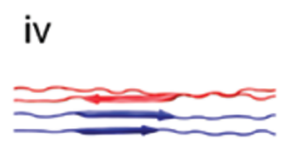

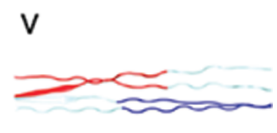

Figure 3. Deformation of the lamin filaments around the crack tip under uniaxial loading at certain strain level. (a) Snapshots of the meshwork geometry near the crack tip at applied strains of (i) $\varepsilon_{y}=17 \%$, (ii) $42 \%$, (iii) $58 \%$, (iv) $160 \%$, (v) $161 \%$. The crack length is $2 a / b=6$ and each filament is colored according to its structural character, as indicated in Figure 1d. (b) Visualization of the molecular structure of the intermediate filament under stretching. The structural character of the filament ahead of the crack tip (marked by a circle in panel a is shown at different strains, corresponding to the strain levels shown in snapshots $\mathrm{i}-\mathrm{v}$ in panel a (the local strain at the filament considered here is $\varepsilon=40 \%, 50 \%, 90 \%, 190 \%$, and $220 \%$ and thus generally larger than the applied strain).

leads to a highly localized stress distribution of $\sigma_{\mathrm{C}} / \sigma_{\mathrm{F}} \approx$ 2.2 (as shown in Figure 1d). We thus obtain $N \gg 1$ (reflecting stiffening) from eq 1 , which agrees with the hypothesis that this case leads to a localized stress distribution. In the large-deformation regime of over $161 \%$ strain and when rupture occurs, single filaments at the crack tip reach the maximum strength as shown in Figure 2a(iv) and break. This localized failure of single filaments at the crack tip results in eventual crack propagation (mediated by sequential breaking of single filaments at the crack tip) without any crack branching or other large-scale failure mechanisms that involve many filaments.

Discussion of These Results. Specific molecular mechanisms that act at different levels of strain control this change of the "local" value of $N$ (in Figure 3a). Lamin filaments initially feature their typical structure as intact coiled-coils (Figure 3b), where clusters of hydrogen bonds are arranged in series and grouped in $\alpha$-helical turns. This geometry facilitates the unfolding of $\alpha$-helical loops after reaching the limit of elastic deformation at 50\%. ${ }^{21}$ The unfolding force until all $\alpha$-helical loops are unfolded is basically constant for long coils, leading to a strong softening behavior (which can be referred to as "ideal elastic-plastic") and thus a hardening exponent of $N \rightarrow 0$. Further loading causes polypeptide strands to be fully unfolded, squeezed together driven by hydrophobic interactions and eventually undergoing a transition into beta-sheets. The structure after this alpha-to-beta transition exhibits a stronger ability to withstand forces because many clusters of hydrogen bonds are loaded in parallel and deform cooperatively, ${ }^{23}$ leading to a severe increase of the force and stiffness as loading is further increased. This results in severe stiffening and is captured in a hardening exponent of $N \gg 1$. An increasing loading causes first sliding and then rupture of the entire filament, leading to a final softening behavior at very large deformation. This analysis shows that the softening and stiffening behavior, described by changes in the "local" hardening exponent $N$, is caused by secondary structure transitions at the molecular level at the nanoscale.

Finally, in order to identify the flaw-tolerance ability of the material for varied defect sizes, we investigate the failure strain $\varepsilon_{\mathrm{F}}$ of the meshwork as a function of the crack length $a$ as shown in Figure 4a. The results depicted in Figure 4a show that the failure strain is largely insensitive to the presence and size of cracks, and that the failure strain approaches a constant value even as the defect size grows. Considering eq 1 we empirically fit the $\varepsilon_{\mathrm{F}}-a$ relation by

$$
\varepsilon_{\mathrm{F}}=\varepsilon_{\mathrm{F} 0}\left(\frac{2 a}{d}\right)^{-1 /(1+N)}
$$

where $\varepsilon_{\mathrm{F} 0}=196 \%$ corresponds to the highest failure strain at $2 a=d$ (for the case where no crack is present, or a perfect meshwork), and $N=7.6$ is the hardening 
a

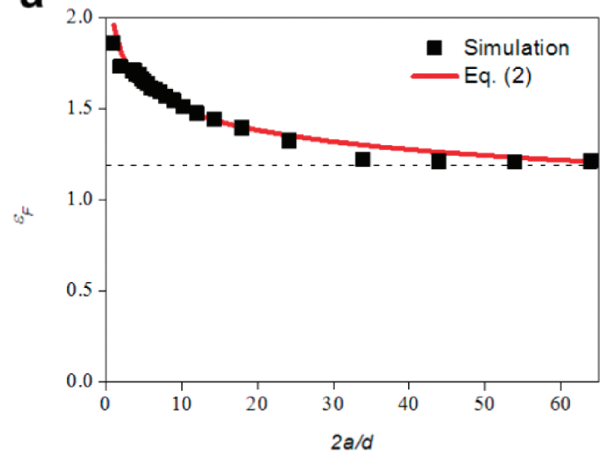

b

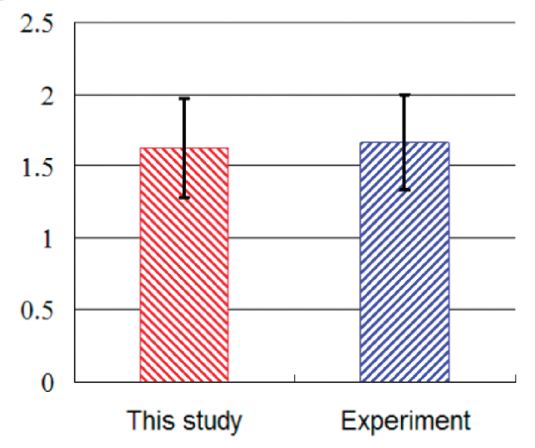

Figure 4. Failure strain $\varepsilon_{\mathrm{F}}$ of the meshwork as a function of initial crack length, here quantified in normalized form (crack length normalized by the lattice constant $d$ ). (a) Failure strain of the meshwork for varied crack sizes. The results show that the failure strain is largely insensitive to the presence and size of cracks, and that the failure strain approaches a constant value of $128 \%$ in the asymptotic limit for very large crack sizes. (b) The comparison of $\varepsilon_{\mathrm{F}}$ between results obtained in this study with a characteristic defect size and the experiment of the axial extensibility of nuclear lamina gel. ${ }^{8}$ The data compares well and provides validation for the results obtained from our computational model.

exponent of nuclear lamina before failure. For the case of biaxial loading with $\varepsilon_{x}=\varepsilon_{y}$, we obtain $\varepsilon_{\mathrm{Fo}}=197 \%$ and $N=6.8$. It is noted that we confirmed that as we change the parameters $h, l$, and $d$ we find the parameters $\left(\varepsilon_{\mathrm{F} 0}, N\right)$ do not change with those variations. The voids of nuclear lamina in experimental studies are observed to be up to on the order of $1.4 \mu \mathrm{m}$ (Supporting Information, Figure $\mathrm{S} 3 \mathrm{~b}$ ), leading to $\varepsilon_{\mathrm{F}}=128 \%$. This analysis explains the phenomenon that the integrity of nuclear lamina is not greatly affected by a single or larger number of clusters (for example, a conglomerate of several nuclear pores), representing a flaw-tolerant material.

Our results also explain recent experimental stretching tests of gels of nuclear lamina, where the material has been found to exhibit a large axial strain before failure in the range of $133 \%-200 \%{ }^{8}$ This agrees with our finding that the failure strain of the nuclear lamina is between two bounds of $128 \%-197 \%$, as shown in Figure 4b. Comparing with the failure strain measured for the entire nuclear envelope (in the range of $50 \%-60 \%$ strain $^{15}$ ), the nuclear lamina is found to be much more extensible, and much greater than the deformation that can be generated by microtubules $\left(<50 \%{ }^{20}\right)$ that impinge on the nuclear envelope. ${ }^{16}$ Therefore, external forces applied outside the cell are likely not sufficient to cause the rupture the nuclear lamina by mechanical signal transduction through microtubules. These results, together with the fact that the integrity of the nuclear lamina only breaks down during mitosis through breakdown processes, suggest that even if there exists a structural flaw, the nuclear lamina is a reliable structure that effectively protects genetic material from extreme external force and deformation applied on the cell, which will not result in rupture of the nuclear lamina unless there is further biochemical modification or mutations that affect its mechanical property to lead to a loss of flaw tolerance. Although the accuracy of this result may be distorted since the gel is composed of lamins in random orientations, it is the only experimental result that quantitatively provides an estimate of the deformation capacity of nuclear laminas. We use this result to carry out the comparison here but are cognizant that future experimentation validations are required to fully understand all the mechanical properties of nuclear laminas. We note that other nuclear lamina models with different geometries have been proposed in other works. ${ }^{12,13,28}$ Since both the in situ and in vitro experiments of somatic nuclear lamina are either not feasible or too difficult to carry out, ${ }^{13}$ it is hard to tell which model is more immediately related to human somatic nuclear lamina. Since our model can in principle be readily adopted to different sets of geometries and mechanical parameters it may be used as a tool to determine which model is more realistic by comparing against future in vivo mechanical test in experiments. It is also noted that within our model we consider imperfections, defects, and heterogeneities simply as crack-like defects, which is a highly simplified representation since the biological model of defects can be more complex. For example, different models of the effect of the nuclear pore complex on the nuclear lamina have been discussed, and those models either consider a nuclear pore complex as a relatively weakly associated deformable unit similar to a void or strongly associated rigid complex similar to a rivet. ${ }^{28}$ Although there is some experimental evidence that nuclear pore complexes act like rivets, ${ }^{28}$ there is also evidence that rupture and breakdown of nuclear envelopes tend to occur around nuclear pore complexes. ${ }^{17}$ The improvement of defect modeling of nuclear pore complexes requires more information of the interaction between the complex and nuclear lamina and has yet to be established.

\section{CONCLUSION}

Using a simple model we identified here the physical basis of the material design of nuclear lamina that 
enables it to withstand extreme mechanical deformation of more than $100 \%$ strain, despite the presence of structural defects. Our study shows that its integrity and strength are not compromised by structural defects such as nuclear pores or other imperfections embedded in nuclear envelopes and that this superior robustness is caused by mechanisms that act at multiple scales. This is facilitated by a combination of softening-stiffening-softening of filament mechanics, which is generated by protein unfolding, an alpha-tobeta transition, and intermolecular sliding under mechanical loading. The robust failure behavior of nuclear lamina is explained theoretically by a change of the stress-strain hardening exponent $N$ as deformation is increased. We derived a general theoretical model that suggests that the critical feature of the dependence of $N(\varepsilon)$ is that $N \rightarrow 0$ (here, for strains up to $90 \%$ ), and $N \gg 1$ for larger strains (here, between $90 \%$ and $176 \%$ ). This particular sequence of nonlinear stress-strain law of filaments facilitates an extremely delocalized deformation at strains of up to $176 \%$ and a highly localized failure once catastrophic breakdown of the material occurs at strains in excess of $176 \%$ strains. The delocalized deformation in the regime up to $176 \%$ vastly increases the energy absorbing capacity, while the localized failure during crack extension provides an important mechanism to ensure localized damage, should it occur. Localized damage is critical for cells to being able to repair defects rather than having to deal with large-scale and widely distributed damage in the nuclear lamina. This suggests that the paradigm of dealing with failure, if it does occur, is that few protein filaments are sacrificed rather than the entire meshwork. As an important barrier to protect genetic material crucial to life, the nuclear lamina must indeed be an extremely reliable structure that can tolerate extreme mechanical conditions. The general mechanism to create such flaw-tolerant behavior revealed here, established through a particular nonlinear stressstrain response of constituting protein filaments realized through a series of molecular mechanisms, may also apply to other natural and synthetic materials, and could enable us to fabricate novel biologically inspired materials with high extensibility, high strength, robustness, and impact resistance. ${ }^{29}$ This opens the possibility that by designing the molecular structure of the material the particular dependence of the hardening exponent $N$ as a function of strain can be achieved. The multiscale approach used here could also enable studies of the mechanism of disease (e.g., rapid aging disease progeria), which is caused by point mutations at the protein level and that affects the mechanical properties of nuclear lamina by making it more brittle.

\section{MATERIALS AND METHODS}

Multiscale Modeling Method. The mesoscopic model used here is set up based on a combination of experimental and full atomistic data. ${ }^{1,12,21,30}$ We use a simple mesoscopic model describing each intermediate filament as a series of beads interacting according to nonlinear interparticle multibody potentials. The model is designed by our desire to develop a simple model to derive generic insight into the mechanical properties and mechanisms. We note that even though such a simple model formulation does not allow us to derive quantitative conclusions for phenomena pertaining to specific types of nuclear laminas, it does enable us to understand universal, generic relationships between underlying molecular mechanisms, resulting nonlinear properties of the material, and the failure behavior of intermediate filament meshworks.

The total energy is given by

$$
E_{\mathrm{x}}=E_{\mathrm{T}}+E_{\mathrm{B}}
$$

The total energy is given by the sum over all pair wise and threebody interactions:

$$
E_{\mathrm{T}}=\sum_{\text {pairs }} \varphi_{\mathrm{T}}(r) \quad \text { and } \quad E_{\mathrm{B}}=\sum_{\text {triplets }} \varphi_{\mathrm{B}}(\theta)
$$

Here we approximate the nonlinear force-extension behavior under tensile loading with a multipolynomial potential model that has been used successfully in the earlier study of deformation of cytoplasm single filament. ${ }^{31}$ The tensile force between two particles (beads as illustrated in Supporting Information, Figure S1c) is described as

$$
F_{\mathrm{T}}(r)=-\frac{\partial \varphi_{\mathrm{T}}(r)}{\partial r}
$$

where

$$
\begin{gathered}
\quad \frac{\partial \varphi_{\mathrm{T}}(r)}{\partial_{r}}=\left[\exp \left(\frac{r-r_{\mathrm{b}}}{r_{\mathrm{b}}} \Xi\right)+1\right]^{-1} \\
\begin{cases}k_{1}\left(r-r_{0}\right) & r<r_{1} \\
R_{1}+k_{2}\left(r-r_{1}\right) & r<r_{2} \\
R_{2}+k_{3}^{1}\left(r-r_{2}\right)+k_{3}^{2}\left(r-r_{2}\right)^{2}+k_{3}^{3}\left(r-r_{2}\right)^{3} & r<r_{3} \\
R_{3} & r \geq r_{3}\end{cases}
\end{gathered}
$$

In eq $6, k_{i}$ and $r_{i}$ are spring constants that derived directly from the force-extension curve of the tension test of full atomic model (as shown in Supporting Information, Figure S1b, with their physical meaning and value defined in Supporting Information). The Fermi-Dirac distribution function introduces two additional parameters $r_{\mathrm{b}}$ and $\Xi .^{32}$ The parameter $r_{\mathrm{b}}$ denotes the critical separation distance for breaking of the filament and the parameter $\Xi$ describes the amount of smoothing around the breaking point (the smaller $\Xi$, the smoother the curve becomes). A similar strategy to model the interatomic potential near rupture has been used in earlier work.

The bending energy (as illustrated in Supporting Information, Figure S1d) is given by

$$
\varphi_{\mathrm{B}}(\theta)=\frac{1}{2} k_{\mathrm{B}}\left(\theta-\theta_{0}\right)^{2}
$$

with $k_{\mathrm{B}}$ relating to the bending stiffness of the intermediate filament $E I$ through $k_{\mathrm{B}}=3 E I / r_{0}$. The parameter $E I$ relates to the intermediate filament persistence length $L_{p}$ through $E I=$ $L_{\mathrm{p}} K_{\mathrm{B}} T$, where $K_{\mathrm{B}}$ is the Boltzmann constant and $T$ is the temperature.

The geometry of the meshwork is obtained from experimental observations of the nuclear lamina of Xenopus oocytes. ${ }^{1}$

The meshwork is composed of two sets of near-orthogonal 
intermediate filaments with a lattice constant of $d=50 \mathrm{~nm}$, and we use $d$ to normalize the crack length, and that value gives the size of defects. We use mesoscopic beads to model the each intermediate filament within the meshwork and use $r_{0}=5 \mathrm{~nm}$, this length is much smaller than the persistence length of the full length filament ( $1 \mathrm{um})$, and it equals to the radius of a full length filament.

Computational Experiments. Calculations are carried out in two steps, first relaxation followed by loading. Relaxation is achieved by heating up the system, then annealing the structure at temperature of $300 \mathrm{~K}$, followed by energy minimization. After relaxation, we keep the system at $300 \mathrm{~K}$ in an NVT ensemble (constant temperature, constant volume, and constant number of particles) and apply loading by displacement boundary conditions (by fixing one single layer of beads near the meshwork boundary and apply the load in normal directions. This setup resembles linear rails with guides clamped to the boundary, resulting in biaxial deformation independently ${ }^{33}$ ), continuously displacing particles in the boundary in a speed of 0.01 $\AA / p s$. Studies with varying loading rates are carried out based on the mesoscopic model of single filaments. By comparing with force-extension curves obtained from atomic model of vimentin intermediate filaments and experimental studies of several classes of intermediate filaments near equilibrium ${ }^{21}$ it is confirmed that the molecular model is validated against experimental data.

Damping. Damping effects are included in the model by considering the energy dissipation of the intermediate filament in motion caused by the viscosity of water environment. We note because of the small character dimension of the intermediate filament, the Reynolds number is $\ll 2300^{34}$ and the drag force is approximately proportional to particle velocity (laminar flow). We use the Stokes' law to measure the drag force by ${ }^{34}$

$$
f_{\text {drag }}=-6 \pi \mu R v
$$

where $\mu=8.6 \times 10^{-4} \mathrm{~Pa} \cdot \mathrm{s}$ is the fluid viscosity constant of water at room temperature, and $R$ is the equivalent spherical radius of the mesoscopic bead, which equals the radius of a sphere of equivalent volume given by ${ }^{35}$

$$
R=\left(\frac{3}{16} b^{2} r_{0}\right)^{1 / 3}
$$

with geometry parameters of the mesoscopic bead given as $R=4.5 \mathrm{~nm}$. The parameter $v$ denotes the relative velocity of particle motion in a continuous viscous fluid.

Acknowledgment. We acknowledge discussions with Kris Dahl (Carnegie Mellon). Support for this research was provided by AFOSR.

Supporting Information Available: Additional information regarding the computational model development, validation, and computing technique and supporting references. This material is available free of charge via the Internet at http:// pubs.acs.org.

\section{REFERENCES AND NOTES}

1. Aebi, U.; Cohn, J.; Buhle, L.; Gerace, L. The Nuclear Lamina Is a Meshwork of Intermediate-Type Filaments. Nature 1986, 323, 560-564.

2. Chang, L.; Shav-Tal, Y.; Trcek, T.; Singer, R. H.; Goldman, R. D. Assembling an Intermediate Filament Network by Dynamic Cotranslation. J. Cell Biol. 2006, 172, 747-758.

3. Herrmann, H.; Bar, H.; Kreplak, L.; Strelkov, S. V.; Aebi, U. Intermediate Filaments: From Cell Architecture to Nanomechanics. Nat. Rev. Mol. Cell Biol. 2007, 8, 562-573.

4. Ishikawa, H.; Bischoff, R.; Holtzer, H. Mitosis and Intermediate-Sized Filaments in Developing Skeletal Muscle. J. Cell Biol. 1968, 38, 538-555.

5. Burke, B.; Stewart, C. L. Life at the Edge: The Nuclear Envelope and Human Disease. Nat. Rev. Mol. Cell Biol. 2002, 3, 575-585.

6. Qin, Z.; Buehler, M. J.; Kreplak, L. A Multiscale Approach to Understand the Mechanobiology of Intermediate Filaments. J. Biomech. 2010, 43, 15-22.
7. Guttinger, S.; Laurell, E.; Kutay, U. Orchestrating Nuclear Envelope Disassembly and Reassembly During Mitosis. Nat. Rev. Mol. Cell Biol. 2009, 10, 178-191.

8. Panorchan, P.; Schafer, B. W.; Wirtz, D.; Tseng, Y. Nuclear Envelope Breakdown Requires Overcoming the Mechanical Integrity of the Nuclear Lamina. J. Biol. Chem. 2004, 279, 43462-43467.

9. Eriksson, M.; Brown, W. T.; Gordon, L. B.; Glynn, M. W.; Singer, J.; Scott, L.; Erdos, M. R.; Robbins, C. M.; Moses, T. Y.; Berglund.; et al. Recurrent de Novo Point Mutations in Lamin a Cause Hutchinson-Gilford Progeria Syndrome. Nature 2003, 423, 293-298.

10. Omary, M. B.; Coulombe, P. A.; McLean, W. H. I. Mechanisms of Disease: Intermediate Filament Proteins and Their Associated Diseases. New Engl. J. Med. 2004, 351, 2087-2100.

11. van der Kooi, A. J.; Bonne, G.; Eymard, B.; Duboc, D.; Talim, B.; Van der Valk, M.; Reiss, P.; Richard, P.; Demay, L.; Merlini, L.; et al. Lamin a/C Mutations with Lipodystrophy, Cardiac Abnormalities, and Muscular Dystrophy. Neurology 2002, 59, 620-623.

12. Goldberg, M. W.; Fiserova, J.; Huttenlauch, I.; Stick, R. A New Model for Nuclear Lamina Organization. Biochem. Soc. Trans. 2008, 36, 1339-1343.

13. Goldberg, M. W.; Huttenlauch, I.; Hutchison, C. J.; Stick, R. Filaments Made from a- and B-Type Lamins Differ in Structure and Organization. J. Cell Sci. 2008, 121, 215-225.

14. Schermelleh, L.; Carlton, P. M.; Haase, S.; Shao, L.; Winoto, L.; Kner, P.; Burke, B.; Cardoso, M. C.; Agard, D. A.; Gustafsson, M. G.; Leonhardt, H.; Sedat, J. W. Subdiffraction Multicolor Imaging of the Nuclear Periphery with 3d Structured Illumination Microscopy. Science 2008, 320, 1332-1336.

15. Dahl, K. N.; Kahn, S. M.; Wilson, K. L.; Discher, D. E. The Nuclear Envelope Lamina Network Has Elasticity and a Compressibility Limit Suggestive of a Molecular Shock Absorber. J Cell Sci 2004, 117, 4779-4786.

16. Beaudouin, J.; Gerlich, D.; Daigle, N.; Eils, R.; Ellenberg, J. Nuclear Envelope Breakdown Proceeds by MicrotubuleInduced Tearing of the Lamina. Cell 2002, 108, 83-96.

17. Djaczenk., W; Starzyk, H.; Rzucidlo, Z. X-ray-Irradiation Induced Changes of Nuclear Membrane of KirkmanRobbins Tumor-Cells. Experientia 1973, 29, 83-84.

18. Munro, T. R. Relative Radiosensitivity of Nucleus and Cytoplasm of Chinese Hamster Fibroblasts. Radiat. Res. 1970, 42, 451-470.

19. Szekely, J. G.; Copps, T. P.; Morash, B. D. Radiation-Induced Invagination of the Nuclear-Envelope. Radiat. Res. 1980, $83,621-632$

20. Janmey, P. A.; Euteneuer, U.; Traub, P.; Schliwa, M. Viscoelastic Properties of Vimentin Compared with Other Filamentous Biopolymer Networks. J. Cell Biol. 1991, 113, 155-160.

21. Qin, Z.; Kreplak, L.; Buehler, M. J. Hierarchical Structure Controls Nanomechanical Properties of Vimentin Intermediate Filaments. PLOS ONE 2009, 4, e7294.

22. Ackbarow, T.; Chen, X.; Keten, S.; Buehler, M. J. Hierarchies, Multiple Energy Barriers, and Robustness Govern the Fracture Mechanics of Alpha-Helical and Beta-Sheet Protein Domains. Proc. Natl. Acad. Sci. U.S.A. 2007, 104, 16410-16415.

23. Qin, Z.; Buehler, M. J. Molecular Dynamics Simulation of the Alpha-Helix to Beta-Sheet Transition in Coiled Protein Filaments: Evidence for a Critical Filament Length Scale. Phys. Rev. Lett. 2010, 104, 198304.

24. Keten, S.; Xu, Z. P.; Ihle, B.; Buehler, M. J. Nanoconfinement Controls Stiffness, Strength and Mechanical Toughness of Beta-Sheet Crystals in Silk. Nat. Mater. 2010, 9, 359-367.

25. Fudge, D. S.; Gardner, K. H.; Forsyth, V. T.; Riekel, C.; Gosline, J. M. The Mechanical Properties of Hydrated Intermediate Filaments: Insights from Hagfish Slime Threads. Biophys. J. 2003, 85, 2015-2027.

26. Rice, J. R.; Rosengren, G. F. Plane Strain Deformation near a Crack Tip in a Power-Law Hardening Material. J. Mech. Phys. Solids 1968, 16, 1-12.

27. Pugno, N.; Carpinteri, A.; Ippolito, M.; Mattoni, A.; Colombo, L. Atomistic Fracture: QFM vs MD. Eng. Fract. Mech. 2008, 75, 1794-1803. 
28. Rowat, A. C.; Foster, L. J.; Nielsen, M. M.; Weiss, M.; Ipsen, J. H. Characterization of the Elastic Properties of the Nuclear Envelope. J. R. Soc. Interface 2005, 2, 63-69.

29. Buehler, M. J.; Yung, Y. C. Deformation and Failure of Protein Materials in Physiologically Extreme Conditions and Disease. Nat. Mater. 2009, 8, 175-188.

30. Xu, Z. P.; Paparcone, R.; Buehler, M. J. Alzheimer's a Beta(1-40) Amyloid Fibrils Feature Size-Dependent Mechanical Properties. Biophys. J. 2010, 98, 2053-2062.

31. Bertaud, J.; Qin, Z.; Buehler, M. J. Intermediate FilamentDeficient Cells Are Mechanically Softer at Large Deformation: A Multiscale Simulation Study. Acta Biomater. 2010, 6, 2457-2466.

32. Buehler, M. J.; Gao, H. J. Dynamical Fracture Instabilities Due to Local Hyperelasticity at Crack Tips. Nature 2006, 439, 307-310.

33. Petersan, P. J.; Deegan, R. D.; Marder, M.; Swinney, H. L. Cracks in Rubber under Tension Exceed the Shear Wave Speed. Phys. Rev. Lett. 2004, 93, 015504.

34. Batchelor, G. K. An Introduction to Fluid Dynamics; 1st Cambridge Mathematical Library ed.; Cambridge University Press: Cambridge, U.K.; New York, 2000.

35. Jennings, B. R.; Parslow, K. Particle-Size MeasurementThe Equivalent Spherical Diameter. Proc. R. Soc. London, Ser. a 1988, 419, 137-149. 